\title{
Maintenance of diet participation in individuals with spinal cord injury: effect on mood and neuropathic pain
}

\author{
David J. Allison ${ }^{1,2} \cdot$ David S. Ditor $^{1,2}$
}

Received: 10 September 2018 / Revised: 12 October 2018 / Accepted: 16 October 2018

(c) International Spinal Cord Society 2018

\begin{abstract}
Study design: One-year follow-up of a randomized clinical trial.

Objectives: Examine voluntary dietary compliance 1 year following the cessation of the intervention and the persistence of accrued benefits related to neuropathic pain and mood.

Setting: Outpatient rehabilitation program within the Niagara region.

Methods: Five individuals (4 female, 1 male; age 50.6 \pm 11.8 years) with chronic SCI (C5-L3; ISNCSCI: A-D; $7-40$ years post injury) who had previously completed a 3-month anti-inflammatory diet were reassessed after 1 year for measures related to dietary compliance, CES-D scores of depression, and NPQ scores of neuropathic pain.

Results: There was a significant reduction in diet compliance at the 1-year follow-up in comparison to the end of the dietary intervention at 3 months $(92.6 \%$ versus $43.0 \%, p<0.01)$. CES-D scores showed a trend toward an increase from 3 months to follow-up (8.0 versus $21.4, p=.10$ ) whereby follow-up CES-D scores were no longer statistically different from baseline $(p=0.74)$. Sensory NPQ scores showed no significant change from 3 months to follow-up $(25.2$ versus $29.1, p=0.42)$ and remained significantly lower than baseline $(p=0.02)$. Affective NPQ scores were significantly increased from 3 months to follow-up (27.7 versus 40.1, $p=0.05)$. Sensitivity NPQ scores showed no significant change from 3 month to follow-up ( 28.2 versus $33.5, p=0.34$ ), but returned to a score that was statistically similar to baseline $(p=0.15)$.

Conclusions: These results emphasize the importance of continued compliance to a diet with anti-inflammatory properties for the purposes of maintaining benefits related to mood and neuropathic pain in individuals with SCI.
\end{abstract}

Sponsorship: This study was supported by the Ontario Neurotrauma Foundation.

\section{Introduction}

Damage to the nervous system following spinal cord injury can result in lasting alterations to nociceptive thresholds and a unique form of pain known as neuropathic pain. Neuropathic pain can be severely debilitating and is estimated to affect between 29 and $75 \%$ of the spinal cord injured population [1]. Further, rates of major depressive disorder (MDD) are substantially higher in this population [2, 3], both as a comorbidity of and independent of neuropathic

David J. Allison

allisodj@mcmaster.ca

1 Department of Kinesiology, Brock University, St Catharines, Ontario L2S 3A1, Canada

2 Brock-Niagara Centre for Health and Well-being, St Catharines, Ontario L2T 1W4, Canada pain. Although each of these disorders have complex and heterogenous etiologies, they both share a common etiological influence related to chronic inflammation. A number of inflammatory mediators have been shown to reduce the nociceptive threshold and produce hyperalgesic effects via both direct and indirect (prostaglandin-dependent) mechanisms [4]. They are also capable of altering the balance of key neuroactive compounds, both centrally and peripherally, by influencing the function of transporters, enzymes, and receptors, which ultimately impact symptoms of depression [5, 6]. As each of these disorders can severely impact one's quality of life [7], and are notoriously difficult to treat $[8,9]$, the exploration of novel treatment strategies is of critical importance.

To this end, our lab performed a randomized controlled trial to explore the efficacy of utilizing an anti-inflammatory diet for the purpose of treating symptoms of neuropathic pain and depression. We found that the implementation of a 3-month anti-inflammatory diet was effective at reducing 
serum concentrations of proinflammatory mediators, and this reduction translated into significant improvements in both neuropathic pain [10] and depression [11]. Further, participants demonstrated excellent adherence to the diet with an average compliance score of $89 \%$.

While these results are encouraging, it was not known whether participants would maintain these dietary habits following the completion of the study. Further, it was unclear if diet-related improvements in depression and neuropathic pain would persist even without continued adherence to the diet. The purpose of this follow-up study was twofold: (a) to determine the degree to which participants remained compliant to the anti-inflammatory diet 1 year following the cessation of the study and (b) to explore the impact this had on symptoms of neuropathic pain and depression.

\section{Methods}

Of the 12 original participants, 5 (4 female, 1 male; age $50.6 \pm 11.8$ years) with chronic SCI (C5-L3; ISNCSCI: A-D; 7-40 years post injury) agreed to participate in the follow-up study. Participant characteristics are summarized in Table 1. All participants had previously completed the aforementioned randomized controlled trial which evaluated the effect of a 3-month anti-inflammatory diet on mood, neuropathic pain, cognition, and somatic nerve function. In brief, all participants in the present study were from the treatment group of a larger original study $(n=20 ; 12$ dieters, all of whom successfully completed the intervention; 8 non-dieting controls) which implemented a diet focused on introducing foods and supplements with established anti-inflammatory properties while eliminating inflammation-inducing foods. This included the removal of various forms of foods such as those with high glycemic indices (e.g., refined sugars), common food intolerances (e.g., cow's milk), and those which negatively influence

Table 1 Participant characteristics

\begin{tabular}{|c|c|c|c|c|c|}
\hline Participant & Sex & Age (y) & $\begin{array}{l}\text { ISNCSCI } \\
\text { score }\end{array}$ & $\begin{array}{l}\text { Level of } \\
\text { injury }\end{array}$ & $\begin{array}{l}\text { Time since } \\
\text { injury (y) }\end{array}$ \\
\hline
\end{tabular}

\begin{tabular}{clllll}
\hline Treatment & & & & & \\
1 & F & 44 & D & C5 & 10 \\
2 & F & 62 & D & L3 & 4 \\
3 & F & 37 & A & T3 & 19 \\
4 & F & 64 & D & C3 & 37 \\
5 & M & 45 & A & T6 & 28 \\
AVE & - & 51.5 & - & - & 12.8 \\
SD & - & 15.3 & - & - & 11.3 \\
\hline
\end{tabular}

ISNCSCI International Standards for Neurological Classification of Spinal Cord Injury cardiovascular health (e.g., hydrogenated oils). Participants were also supplied with daily supplements with antiinflammatory properties including: omega-3 (Now Ultra omega-3), chlorella (Now chlorella), antioxidants (CanPrev antioxidant network), curcumin (AOR Inflanox), and a vegetable-based protein powder (Progressive Vegessential).

Following the completion of the RCT, participants were neither encouraged, nor discouraged, to continue with the diet. Supplements were no longer provided to participants free of charge, and weekly support phone calls were stopped. Participants were not informed of the 1-year follow-up study. Follow-up measures included diet compliance, mood, and neuropathic pain.

Diet compliance was assessed via a detailed analysis of a 7-day diet record completed by each participant. Food intake was assessed using the The Food Processor (ESHA Inc. 2014, version 10.14.2, Salem, OR). All food items were categorized as "foods to consume", "foods to avoid", or "neutral foods" based on the parameters of the diet (as previously described [11]) and categorized by size in accordance with Canadas Food Guide. A percent compliance score was calculated as the ratio of servings of foods to consume over the total servings of food (avoid + consume) multiplied by 100 .

For the assessment of mood, participants completed the Center for Epidemiological Studies Depression Scale (CES-D). This questionnaire consists of 20 items related to depression (e.g., I had crying spells), whereby participants are asked to score how often they experienced a particular item over the past 7 days. Each item is scored on a 4-point scale including "rarely or none of the time (less than 1 day)", "some or a little of the time (1-2 days)", "occasionally or a moderate amount of the time (3-4 days)", or "most or all of the time (5-7 days)". Scores for each item range from 0 to 3 and are totaled for a final score ranging between 0 and 60 . A score of 16 points or greater suggests the presence of depressive symptoms.

Neuropathic pain was assessed via the Neuropathic Pain Questionnaire (NPQ) [12]. This questionnaire consists of 32 questions pertaining to three unique categories of neuropathic pain including sensory items $(n=18)$, affective items $(n=8)$, and sensitivity items $(n=6)$. Sensory items related to the specific type and severity of pain (e.g., burning pain), affective items related to the how the pain affected the participant in daily life (e.g., how unpleasant is your usual pain?), and sensitivity items related to how different stimuli influenced pain (e.g., increased pain due to heat). Each item was ranked on a scale from 0 to 100 whereby higher scores indicated worse pain. An average score for each category was then calculated.

One-way repeated measures ANOVA was performed to investigate possible changes in diet compliance, mood and neuropathic pain between baseline, 1-month, 3-months, and 
1-year follow-up. Post hoc $t$-tests were performed as necessary to compare means between time points. These data are expressed as means \pm standard deviations. This study received ethical approval from the Brock University Research Ethics Board and all participants gave informed written consent prior to the administration of questionnaires.

\section{Results}

\section{Summary of findings from original anti- inflammatory diet}

In the original 3-month anti-inflammatory diet study $(n=$ $20)$, participants from the intervention group $(n=12)$ demonstrated a diet compliance score of $89 \%$. There was a significant group $\times$ time interaction for CES-D score $(p=$ 0.01 ) and post hoc analysis showed a significant reduction in CES-D score in the treatment group from baseline to 3 months $(p<0.01)$, but no significant change in the control group $(p=0.74)$. There was a significant group $\times$ time interaction for the sensory component of the NPQ $(p<0.01)$ and post hoc analysis showed a significant reduction in sensory NPQ score in the treatment group from baseline to 3 months $(p=0.01)$, but no significant change in the control group $(p=0.21)$.

\section{Diet compliance}

A repeated measures analysis of variance (ANOVA) performed on the five members who completed the follow-up testing session demonstrated that diet compliance differed significantly between time points $(\mathrm{F}(3,12)=59.4, p<0.01$, Cohens $d=7.7)$. Post hoc analysis revealed that scores of diet compliance increased significantly from baseline to 1 month $(29.9 \%$ versus $93.8 \%, p<0.01)$ as well as from baseline to 3 months $(29.9 \%$ versus $92.6 \%, p<0.01)$. There was also a significant reduction in scores from 3 months to follow-up $(92.6 \%$ versus $43.0 \%, p<0.01)$ whereby followup diet compliance scores were no longer statistically different from baseline $(p=0.18)$. Raw data for compliance scores are available as an additional file.

\section{Mood}

A repeated measures ANOVA performed on the five members who completed the follow-up testing session demonstrated that CES-D scores differed significantly between time points $(\mathrm{F}(3,12)=4.9, p=0.02$, Cohen $d=$ 2.2). Post hoc analysis revealed that CES-D scores showed a trend toward reduction from baseline to 1 month (20.6 versus 8.8, $p=0.06$ ) and a significant reduction from baseline to 3 months (20.6 versus $8.0, p=0.04)$. There was also a trend toward an increase from 3 months to follow-up (8.0 versus 21.4, $p=0.10$ ) whereby follow-up CES-D scores were no longer statistically different from baseline $(p=0.74)$. Raw data for CES-D scores are available as an additional file.

\section{Neuropathic pain}

A repeated measures ANOVA was performed on each category of the NPQ for the five members who completed the follow-up testing session. Sensory scores differed significantly between time points $(\mathrm{F}(3,12)=6.9, p<0.01$, Cohens $d=2.8$ ). Post hoc analysis revealed that sensory scores were significantly reduced from baseline to 1 month (49.1 versus $33.3, p<0.01$ ) as well as from baseline to 3 months (49.1 versus $25.2, p=0.01)$. There was no significant change in sensory scores from 3 month to follow-up ( 25.2 versus $29.1, p=0.42$ ). Sensory scores remained significantly lower than baseline at follow-up $(p=0.02)$. Affective scores differed significantly between time points $(\mathrm{F}(3,12)=6.5, p<0.01$, Cohens $d=2.5)$. Post hoc analysis revealed that affective scores were significantly reduced from baseline to 1 month (50.9 versus $33.3, p=0.05)$ as well as from baseline to 3 months (50.9 versus $27.7, p<$ $0.01)$. There was also a significant increase in scores from 3 months to follow-up (27.7 versus $40.1, p=0.05$ ) whereby follow-up affective scores were no longer statistically different from baseline $(p=0.24)$. Sensitivity scores differed significantly between time points $(\mathrm{F}(3,12)=3.8, p=0.04$, Cohens $d=1.9$ ). Post hoc analysis revealed that sensitivity scores were not significantly reduced from baseline to 1 month (48.7 versus $34.6, p=0.12$ ) but were significantly reduced from baseline to 3 months (48.7 versus $28.2, p=$ $0.02)$. There was no significant change in sensitivity scores from 3 month to follow-up (28.2 versus $33.5, p=0.34$ ), however, follow-up scores were not significantly lower than baseline $(p=0.15)$. Raw data for NPQ scores are available as an additional file.

\section{Discussion}

Results from this follow-up study demonstrated that participants did not maintain the dietary habits adopted during the 3-month intervention. This was shown by a dramatic reduction in compliance scores among the five participants included in the follow-up, whereby scores fell from $92.6 \%$ at the end of the 3-month intervention to $43.0 \%$ at 1 -year post. Interestingly, mood scores followed a similar trend whereby the significant improvement observed at 3 months (CES-D scores suggesting no depression) returned to baseline by 1year post (CES-D scores once again suggesting depression). With regard to neuropathic pain, sensitivity and affective 
scores showed similar trends to that of mood, whereby each returned to scores that were no longer statistically different from baseline. An unexpected finding was that the significant improvement observed in sensory pain at the end of the intervention was maintained by the 1-year follow-up period. Although purely speculative, this finding could be explained by a possible alteration in the pain phenotype. Evidence suggests that the inflammatory influence on pain may not be limited to an increased neuronal expression of ion channels and receptors resulting in the abnormal generation of nociceptive action potentials. It has been proposed that inflammatory mediators may also influence gene expression by nociceptors, resulting in a chronically hyperexcitable pain phenotype [13]. In this respect, a chronic reduction in inflammation may be expected to cause a shift to a hypo-excitable phenotype, thereby helping to promote prolonged improvements in pain which persisted beyond the intervention period.

The fact that the improvements in neuropathic pain and mood were lost (with the exception of sensory scores) when participants no longer complied to the diet, emphasizes the need for its continued practice. It could be argued that the large reduction in compliance scores demonstrates that adherence to such drastic dietary alterations are not practical for long-term use. It may be possible that a number of potential barriers and/or lack of facilitators may have contributed to the large reduction in compliance at 1-year post intervention. Barriers such as those related to high food and supplement costs, a greater effort required for food preparation and meal planning, difficulty following guidelines when eating away from home at restaurants or social events, or simply the will power needed to avoid foods not allowed on the diet could each contribute to low compliance scores. The barriers and facilitators which influenced eating habits during and following the study have been explored in a recent publication by our lab [14].

Despite the apparent difficulties in maintaining dietary habits long-term, the fact that a large reduction in inflammation was achieved in the original study, despite less than optimal compliance scores, is encouraging as may suggest that a less strict, and therefore easier to maintain, diet may still produce favorable results. It may be possible to achieve long-term diet adherence by reducing the necessary compliance (e.g., allowing "cheat" meals) or reducing the number of restricted foods (e.g., those with less evidence to suggest a proinflammatory influence), while still attaining anti-inflammatory benefits.

The current follow-up study is limited by a lack of biomarker data and small sample size. It does, however, demonstrate a regression whereby much of the benefit accrued during the intervention, related to depression and neuropathic pain, were lost following the cessation of the diet intervention. Future larger-scale studies will be required to further explore which aspects of the diet are most effective in reducing inflammation, and what degree of compliance is necessary in order to maintain antiinflammatory benefits.

\section{Conclusion}

In conclusion, 1 year following the cessation of an antiinflammatory diet intervention, our participants showed poor dietary compliance scores. Additionally, the improvement in mood and two of the three components of neuropathic pain observed at the end of the initial intervention were lost at follow-up. These results emphasize the importance of continued compliance to a diet with antiinflammatory properties and stress the need for further exploration of the barriers which may impede the adoption of long-term dietary practices.

Acknowledgements This study was supported by the Ontario Neurotrauma Foundation.

\section{Compliance with ethical standards}

Conflict of interest The authors declare that they have no conflict of interest.

\section{References}

1. Siddall PJ, McClelland JM, Rutkowski SB, Cousins MJ. A longitudinal study of the prevalence and characteristics of pain in the first 5 years following spinal cord injury. Pain. 2003;103:249-57. https://doi.org/10.1016/S0304-3959(02)00452-9

2. Craig A, Tran Y, Middleton J. Psychological morbidity and spinal cord injury: a systematic review. Spinal Cord. 2009;47:108-14. https://doi.org/10.1038/sc.2008.115

3. Kennedy P, Rogers BA. Anxiety and depression after spinal cord injury: a longitudinal analysis. Arch Phys Med Rehabil. 2000;81:932-7. https://doi.org/10.1053/apmr.2000.5580

4. Woolf CJ, Mannion RJ. Neuropathic pain: aetiology, symptoms, mechanisms, and management. Lancet. 1999;353:1959-64. https://doi.org/10.1016/S0140-6736(99)01307-0

5. Allison DJ, Ditor DS. The common inflammatory etiology of depression and cognitive impairment: a therapeutic target. J Neuroinflamm. 2014;11:151 https://doi.org/10.1186/s12974-0140151-1

6. Watkins LR, Maier SF, Goehler LE. Cytokine-to-brain communication: a review \& analysis of alternative mechanisms. Life Sci. 1995;57:1011-26.

7. Tulsky DS, Kisala PA, Victorson D, Tate DG, Heinemann AW, Charlifue S, et al. Overview of the Spinal Cord Injury--Quality of Life (SCI-QOL) measurement system. J Spinal Cord Med. 2015;38:257-69. https://doi.org/10.1179/2045772315Y.0000000023

8. Hagen EM, Rekand T. Management of neuropathic pain associated with spinal cord injury. Pain Ther. 2015;4:51-65. https:// doi.org/10.1007/s40122-015-0033-y

9. Penn E, Tracy DK. The drugs don't work? Antidepressants and the current and future pharmacological management of 
depression. Ther Adv Psychopharmacol. 2012;2:179-88. https:// doi.org/10.1177/2045125312445469

10. Allison DJ, Thomas A, Beaudry K, Ditor DS. Targeting inflammation as a treatment modality for neuropathic pain in spinal cord injury: a randomized clinical trial. J Neuroinflamm. 2016;13:152 https://doi.org/10.1186/s12974-016-0625-4

11. Allison DJ, Ditor DS. Targeting inflammation to influence mood following spinal cord injury: a randomized clinical trial. J Neuroinflamm. 2015;12:204 https://doi.org/10.1186/s12974015-0425-2
12. Krause SJ, Backonja M-M. Development of a neuropathic pain questionnaire. Clin J Pain. 2003;19:306-14.

13. Miller RJ, Jung H, Bhangoo SK, White FA. Cytokine and chemokine regulation of sensory neuron function. Handb Exp Pharmacol. 2009;194. https://doi.org/10.1007/978-3-540-79090-7.

14. Bailey KA, Lenz K, Allison DJ, Ditor DS. Barriers and facilitators to adhering to an anti-inflammatory diet for individuals with spinal cord injuries. Heal Psychol Open. 2018;5:1-11. https://doi.org/10. 1177/2055102918798732 